\title{
Redefining the clinical gaze
}

\section{Peter Lachman}

\section{Correspondence to}

Dr P Lachman, Great Ormond

Street Hospital NHS Foundation

Trust, Great Ormond Street,

London WC1N 3JH, UK;

Peter.lachman@gosh.nhs.uk

Received 16 July 2013

Accepted 16 July 2013

Published Online First

2 August 2013

\section{CLinked}

- http://dx.doi.org/10.1136/ bmjqs-2012-001467

To cite: Lachman P. BMJ

Qual Saf 2013;22:888-890.
Michel Foucault, in The birth of the clinic, ${ }^{1}$ introduced the concept of the clinical gaze in a wide ranging examination of the emergence of modern medicine. He proposed that the development of the clinical gaze, in which disease was viewed from a new perspective, changed the course of medicine and healthcare. "It is as if for the first time for thousands of years, doctors, free at last of theories and chimeras, agreed to approach the object of their experience with the purity of an unprejudiced gaze." ${ }^{1}$ The new modern scientific medicine resulted in the perceptions of the doctor becoming more important than the views of the patient. The concept of the clinical gaze is a useful concept when one reviews the appearance of the patient safety movement over the past 20 years. The gaze of clinical medicine is transforming, as the safety of patients and person centred care are becoming essential requirements of care. The realisation that healthcare can be made safer is a recent phenomenon and achieving this goal remains a formidable challenge. ${ }^{2}$

In 2009, Leape et al assessed the state of the patient safety movement and the message was stark: "While efforts to improve patient safety have proliferated during the past decade, progress toward improvement has been frustratingly slow. Some of this lack of progress may be attributable to the persistence of a medical ethos, institutionalized in the hierarchical structure of academic medicine and healthcare organizations, that discourages teamwork and transparency and undermines the establishment of clear systems of accountability for safe care." ${ }^{3}$ The development of the clinical gaze changed the relationship between clinicians and patients by emphasising the interpretation of the doctors over that of the patient. The resulting changes in the way health and disease were perceived benefited patients in many ways. One unfortunate consequence, however, has been the medicalisation of health and less patient centred care. $^{4}$
Another problem that has emerged is that the clinical gaze has not been a unified one and the conflicting gazes of differing health professions have resulted in decreased teamwork, which impacts on care coordination. The medical ethos, which emerged from the concept of the clinical gaze, has yet to be transformed. What is needed is a new gaze-perhaps the gaze of patient safety that encompasses the way we organise and deliver healthcare and that works to integrate care. $^{5} 6$

The quest for the safe delivery of healthcare is even more pressing, as healthcare systems are subject to perceived and actual failures, and evidenced by the recent reports in England. ${ }^{78}$ Both providers and regulators of healthcare have been unable to guarantee safe care. The numerous conclusions and recommendations, together with the reaction from the regulators and politicians, suggest that the solution for patient safety is for clinicians to work harder and to be held to account for failures; and for regulators to inspect more frequently and vigorously. While these may have a place in solving the problem, the key is to consider what is really needed to deliver safe and reliable care. No one in healthcare can assume that safety has become the way of life. A radical rethink of what we do is needed, but solutions are elusive. As one considers the culture change that is required, one could become disillusioned at the slow progress of the patient safety movement within healthcare.

The five key factors that were identified by Leape $e t a l^{3}$ remain unfulfilled in most organisations-namely, transparency, integration of care, consumer engagement, joy and meaning in work, and reform of medical education. Four years on, success in any of the five is patchy and progress is difficult to demonstrate in healthcare systems. Wachter gives progress only a ' $\mathrm{B}-$ ' ' in his analysis of what has been achieved. ${ }^{9}$ In a review of the literature on safety cultures, Weaver $e t ~ a l^{10}$ identified a number of interventions thought to be of 
benefit, although levels of evidence were variable. These included team training and communication tools, executive walk rounds, interdisciplinary rounding and unit based safety programmes, which were found to be most effective. Yet the consistent implementation of these solutions remains problematic.

A criticism of the patient safety movement has been the paucity of science underpinning proposed interventions. A recent technical report commissioned by the US Agency for Healthcare Research and Quality compiles the evidence supporting widely recommended patient safety practices. ${ }^{11}$ Ten interventions are suggested to be of importance and as having clear evidence that they work if implemented. Many others have a sound basis and could be considered. This provides the 'what' needs to be done but the 'how' to implement remains the challenge.

Perhaps one needs to go further than just looking to decrease harm by error reduction and improved processes. The concept of taking a systems approach to patient safety and understanding the underlying factors is now accepted as the foundation for patient safety. ${ }^{12}$ Adding concepts of human factors provides further support to the need to have a holistic, systems based approach to safer care. ${ }^{13}$ Yet despite the increasing understanding of the theory of patient safety and what is needed, we remain unable to materially improve, unless the key factors identified by Leape et $a l^{3}$ are addressed.

The very construct of healthcare systems, based on a hierarchical medical education with a training system still firmly rooted in the medieval guild system, will need to transform into one in which patient safety is core to education and not an optional extra. The ongoing reluctance to be fully transparent and the marginalisation of quality and patient safety in the academic literature, as well as in medical schools, will need to be revisited. Violations, which often remain the norm in the name of professional autonomy, and without sanction, will require a redefinition of the concept of medical professionalism. ${ }^{14}$ Amalberti et $a l^{15}$ highlighted the need to move from autonomy to teamwork with a redefinition of roles; yet autonomy remains paramount among most physicians.

The enduring preference for individual autonomy could conceivably reflect the historical focus on technical aspects of care. Technical advances in the diagnosis and treatment of disease may have left safety and compassionate care behind, but they have generated incredible successes. In paediatrics this has been dramatic. Premature infants who died at birth are now kept alive. Children with cancers such as leukaemia live a near normal life expectancy, and many previously chronically disabling diseases such as juvenile arthritis and diabetes are now managed in a way that allows normal development into adulthood. Yet nothing comes without a price. There are the children who now live with increasing complexity in their clinical conditions, and who are most likely to be exposed to harm. The same risk applies to all those exposed to healthcare.

Healthcare professionals now need to focus on redesigning and recalibrating care so that compassion and safety are at the core of care. Healthcare designed for safety integrates human and environmental factors, and then works on improving the processes. Clinicians cannot improve care by working harder at processes without addressing the core philosophical foundations of healthcare.

High reliability and resilience have become the goals of organisations searching for safer systemsthat is, to provide the evidence based care that is needed and required the first time every time. ${ }^{16} 17$ Other industries have introduced reliable and resilient systems ${ }^{18}$ so it should be possible to do so in healthcare. The application of reliability theory in the delivery of healthcare has had variable results. The key ingredients are the concepts of mindfulness ${ }^{19} 20$ and situational awareness. ${ }^{21}$ These allow the clinical team to integrate the different clinical gazes into a shared gaze, taking into account different perspectives.

The redesign of healthcare to incorporate patient safety has been developed in the work on 'microsystems', ${ }^{22}$ which has shown that it is possible to fundamentally change the way the system works. There are several examples of excellence from centres that have demonstrated that one can develop a system in which resilience and designing for safety become the norm rather than the exception. ${ }^{23}$ The theory on patient safety is now well documented but not widely understood in the frontline. ${ }^{24}$

The paper by Goldenhar et $a l^{25}$ provides insight and understanding into some of the mechanisms of safety culture change and the challenges that are posed as these are put into action. They note that huddles or briefings are not a new phenomenon, but the mechanism has not been fully understood. They suggest that there must be a change in the way we approach the solutions for safety, and that by understanding what happens when the processes change perhaps one can fundamentally redesign the service. Huddles are relatively simple interventions with several complex elements that have a major benefit.

The approach discussed by Goldenhar et $a l^{25}$ goes someway to address the challenge set out by Leape et $a l^{3}$ in that there is a change in the medical ethos, a flattening of hierarchy, promotion of teamwork and clear lines of accountability and responsibility in a transparent system of care. The work undertaken is therefore relevant for all of those involved in healthcare and provides an insight into how to address the deficiencies of the current construct in healthcare design. It is a sufficiently small test of change that can be implemented without much cost. Besides the benefits of improving safety, there is the additional 
advantage that huddles offer a way to improve flow and deliver greater value. Huddles may increase clinical mindfulness and decrease cognitive bias, leading to the reduction of missed and delayed diagnosis. This potential benefit warrants further research. ${ }^{26}$ The development of a team approach to the huddle allows the fragmentation of care to be remedied and for a rebalancing of the clinical gaze, which was clinician focused, to one which is person centred with a collegial approach to healthcare. It makes integrated care a distinct reality and offers promise for the future.

Challenges still remain in determining how the concepts of the huddle can be spread throughout healthcare, given the time it took to implement in a high performing organisation. Distributive leadership is an essential ingredient. The empowerment of the frontline to introduce versions of the huddle is the next step. The introduction of the huddle offers a way of redefining clinical care, from the clinical gaze in which we treat disease into the patient safety and quality gaze in which the needs and wants of patients are the core of all that we do.

Acknowledgements I thank David Vaughan and John Fitzsimons for their review and critique of the paper.

Competing interests None.

Provenance and peer review Commissioned; not externally peer reviewed.

\section{REFERENCES}

1 Michel Foucault. The birth of the clinic: archaeology of medical perception. London: Routledge, 1973.

2 Kohn LT, Corrigan JM, Donaldson MS, eds. To err is humanbuilding a safer health system. Washington, DC: National Academies Press, 2000.

3 Leape L, Berwick D, Clancy C, et al. Transforming healthcare: a safety imperative. Qual Saf Health Care 2009;18:1-6.

4 Illich I. Limits to medicine. Medical nemesis: the expropriation of health. London: Marion Boyars, 1974.

5 Bergeson SC, Dean JD. A systems approach to patient-centered care. JAMA 2006;296:2848-51.

6 Ham C, Walsh N. Making integrated care happen at scale and pace. Lessons from experience. London: Kings Fund, 2013. http://www.kingsfund.org.uk/sites/files/kf/field/field_ publication_file/making-integrated-care-happen-kingsfundmar13.pdf (accessed 2 Jul 2013).

7 Report of the Mid Staffordshire NHS Foundation Trust public inquiry (RobertFrancis, chair). Staffordshire. UK: Mid Staffordshire NHS Foundation Trust, February 2013.
8 The Care Quality Commission Project Ambrose. 2013. http:// www.cqc.org.uk/sites/default/files/media/documents/grant_ thornton_uk_llp_morecambe_bay.pdf (accessed 2 Jul 2013).

9 Wachter RM. Patient safety at ten: unmistakable progress, troubling gaps. Health Affairs 2010;29:165-73.

10 Weaver SJ, Lubomksi LH, Wilson RF, et al. Promoting a culture of safety as a patient safety strategy. Ann Intern Med 2013;158:369-74.

11 Shekelle PG, Wachter RM, Pronovost PJ, et al. Making health care safer II: an updated critical analysis of the evidence for patient safety practices. (Prepared by the Southern California-RAND Evidence-based Practice Center under contract HHSA290200710062I.) Rockville, MD: Agency for Healthcare Research and Quality. 2013. http://www.ahrq.gov/ research/findings/evidence-basedreports/ptsafetyuptp.html (accessed 2 Jul 2013).

12 Vincent C, Moorthy K, Sarker SK, et al. Systems approaches to surgical quality and safety: from concept to measurement. Ann Surg 2004;239:475-282.

13 Carayon P, Schoofs Hundt A, Karsh B-T, et al. Work system design for patient safety: the SEIPS model. Qual Saf Health Care 2006;15(Suppl 1):i50-8.

14 Amalberti R, Vincent C, de Saint Maurice G. Violations and migrations in health care: a framework for understanding and management. Qual Saf Health Care 2006;15(Suppl I):i66-71.

15 Amalberti R, Auroy Y, Berwick D, et al. Five system barriers to achieving ultrasafe health care. Ann Intern Med 2005;142:756-64.

16 Wilson KA, Burke CS, Priest HA, et al. Promoting health care safety through training high reliability teams. Qual Saf Health Care 2005; 14:303-9.

17 Hollnagel E, JBraithwaite J, Wears RL, eds. Resilient health care. London: Ashgate, September 2013.

18 Weick K, Sutcliffe K. Managing the unexpected: resilient performance in an age of uncertainty. 2nd edn. San Francisco: Jossey-Bass, 2007.

19 Ludwig DS, Kabat-Zinn J. Mindfulness in medicine. JAMA 2008;300:1350-2.

20 Epstein RM. Mindful practice. JAMA 1999;282:833-9.

21 Endsley M, Garland D. Situation awareness: analysis and measurement. Mahwah, NJ: Lawrence Erlbaum Associates, 2000.

22 Mohr JJ, Barach P, Cravero JP, et al. Microsystems in health care: Part 6. Designing patient safety into the microsystem. Jt Comm J Qual Saf 2003;29:401-8.

23 Bohmer RMJ. The four habits of high-value health care organizations. N Engl J Med 2011;36:2045-7.

24 Vincent $\mathrm{C}$, Burnett S, Carthey J. The measurement and monitoring of safety. London: The Health Foundation, 2013.

25 Goldenhar LM, Brady PW, Sutcliffe KM, et al. Huddling for high reliability and situation awareness. Qual Saf 2013;22: 899-906.

26 Croskerry P. From mindless to mindful practice-cognitive bias and clinical decision making. N Engl J Med 2013;368:2445-8. 